\title{
Systemic Lupus Erythematosus Simulating Kikuchi Fujimoto's Disease: A Case Report
}

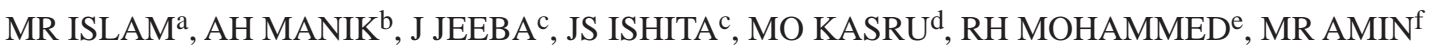

\begin{abstract}
Summary:
Kikuchi Fujimoto's disease (KFD) is a rare, immunemediated, self-limiting disorder with unique histopathological features. KFD is usually seen in young Asian females; however, cases have been reported throughout the world and in all ethnicities. It has been recognized that there is a rare association between Systemic Lupus Erythematosus (SLE) and KFD via sporadic case reports. The exact pathophysiological relationship between these two diseases is still unclear. We report a case of a young Asian female who presented with persistent fever
\end{abstract}

\section{Introduction:}

To the clinician, systemic lupus erythematosus is important as because it is potentially fatal disease that is easily confused with many other disorders. Pathogenic antibodies are produced against the components of the cell nucleus. The abnormal immune response results in target tissue injury, and the ensuing inflammatory reaction deranges various organ functions. Nearly every organ system may be involved in the disease course. It may present initially with non-specific symptoms or atypical manifestations, so the diagnosis may present a considerable

challenge at the early stages ${ }^{1-3}$.On the other hand, Kikuchi Fujimoto's disease (KFD) is a rare, immunemediated, typically self-limiting disease first described

a. Dr. Mohammad Rafiqul Islam, Assistant Professor of Medicine, Dhaka Medical College

b. Dr. Abul Hayat Manik, Medical Officer, Department of Medicine, Dhaka Medical College

c. Dr. Jannat Jeeba, Jenifar Sharmin Ishita, Internee Doctor, Department of Medicine, Dhaka Medical College

d. Dr. Mohammod Omar Kasru, Assistant Registrar, Department of Medicine, Dhaka Medical College

e. Dr. Rakib Hasan Mohammed, Registrar, Department of Medicine, Dhaka Medical College

f. Dr. Md. Robed Amin, Associate Professor of Medicine, Dhaka Medical College

Address of Correspondence: Mohammad Rafiqul Islam. Flat -2B, House-16. Road: 5, Block-B, Banasree, Rampura. Dhaka. Emaildrrafiq73@yahoo.com. Cell- 01753199796. 01921854213

Received: 20 October, 2013

Accepted: 8 July, 2014 followed by development of lymphadenopathy and was diagnosed as Kikuchi Fujimoto's disease based on lymph node biopsy. Although an SLE workup was done and she initially did not meet the American Rheumatology Association (ARA) diagnostic criteria for lupus.The lymph node biopsy did not show typical features of SLE. At last criteria of SLE became obvious with time and case was diagnosed as SLE.

Key words: Kikuchi-Fujimoto, Lymphadenopathy, Pyrexia, SLE.

(J Banagladesh Coll Phys Surg 2014; 32: 231-234)

in Japan in $1972{ }^{4}$, 5 . Clinically, patients present with persistent fever and lymphadenopathy which may be painful; cervical lymph nodes are most commonly involved. Other symptoms such as fatigue, night sweats, nausea and vomiting, weight loss, arthralgia, and a variety of cutaneous lesions are also reported ${ }^{6,7}$. Laboratory findings are nonspecific including elevated erythrocyte sedimentation rate (ESR), neutropenia, lymphocytosis, mildly elevated transaminase, and elevated lactate dehydrogenase (LDH) ${ }^{6-8}$.Diagnosis is made via lymph node biopsy with a histopathological finding characterized by a histiocytic necrotizing lymphadenitis without granulocytic infiltrate ${ }^{6}$. The association of KFD and SLE is described in some studies 9, 10 . It is also speculated that KFD may be one of the manifestations of SLE ${ }^{10}$. Here we are presenting a case where we got dilemma initially between kikuchi's disease and SLE.

\section{Case Report:}

A 23 years female medical student of Dhaka Medical College, presented with high grade continued fever for 6 days .There was no focal symptom except mild headache and anorexia. On query, she did not give any history of travel to any malaria endemic zone, contact with tuberculosis patient and exposure to any medication. On general examination her temperature $102^{\circ} \mathrm{F}$, pulse 114 /minute, blood pressure $80 / 60 \mathrm{~mm}$ of $\mathrm{Hg}$ and mild anemia was present. On systemic examination there was nothing significant. She was admitted to Dhaka Medical College Hospital with 
suspicion of possible viral fever. Some investigations were sent immediately. Patient was admitted to hospital with advice to take plenty of fluid intake and Tab.Paracetamol $500 \mathrm{mg}$ three times daily.

On next day, her investigations reports were S. ALT- 45 U/L, S. AST- 90 U/L. Complete blood count reveals hemoglobin- $10 \mathrm{gm} / \mathrm{dl}$, total count of white blood cell$10.6 \times 10^{3} / \mathrm{mm}^{3}$, ESR $100 \mathrm{~mm}$ in $1^{\text {st }}$ hour, $\mathrm{N}-74 \%$, L21\%, E- $0.4 \%$. Dengue NS1 antigen was also negative. Other investigations were within normal limit.

On $4^{\text {th }}$ day of her admission, there was no sign of remission of fever. Meticulous physical examination was done and there was nothing significant. Empirically Inj. Ceftriaxon 2 gm twice daily was started. Spiking fever and constitutional symptoms persisted. On $10^{\text {th }}$ day, we started Inj. Meropenem $1 \mathrm{gm}$ three times daily keeping Ceftriaxon 2 gm twice daily continued. On $11^{\text {th }}$ day of her admission, detailed examination done again. This time few palpable lymph nodes were present in the anterior and posterior cervical chain, left supra clavicle region and also bilateral inguinal region. Lymph nodes were discrete, tender, free from underlying and overlying structure and largest one was $1.5 \times 1.5$ centimeter in left anterior cervical chain. We started a third antibiotic with Moxifloxacin $400 \mathrm{mg}$ through intravenous route daily as her fever was not responding to previous regime. Some investigations were sent from day 9 to day11.

Reports of investigations were as follows, $\mathrm{Hb}-7.9 \mathrm{gm} /$ $\mathrm{dl}$, total count- $3.4610^{3} / \mathrm{mm}^{3}$, N-84\%, L-15\%, M-0.6\%, platelet count- $14610^{3} / \mathrm{mm}^{3}$, ESR-100 mm in 1st hour, CRP-23.7 mg/L and peripheral blood film revealed normocytic normochromic anemia with moderate rolouex formation. Blood for malarial parasite and triple antigen were negative. Urine R/M/E and C/S were also normal. Mantoux test result after 72 hours was $4 \mathrm{~mm}$. Ultrasonography of whole abdomen revealed mild hepatomegaly, cholelithiasis, enlarged ovaries but no ascites and pleural effusion. FNAC of left supraclavicular lymph node revealed focal aggregation of epithelioid cells. Background shows neutrophil,lymphocyte. No malignancy seen. Features suggestive of granulomatous inflammation (Fig-1). ANA (Indirect immunofluresence on He-2 ell) showed strongly positive, pattern- Speckled variety. Serum ferritin was $5689.09 \mu \mathrm{gm} / \mathrm{L}$.

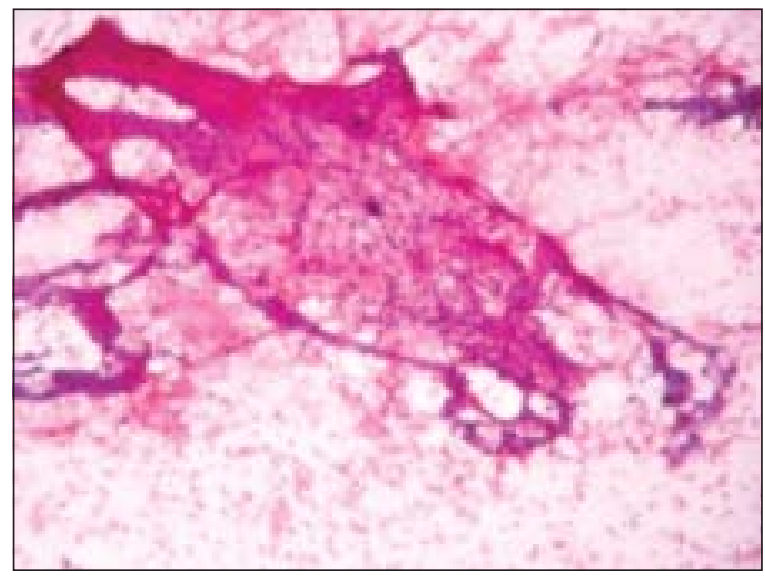

Fig.-1: FNAC of Lymph Node showing granulomatous inflammation.

We started category one anti TB regimen on the background of fever, lymphadenopathy, high TB prevalence in this subcontinent and finding of lymph node FNAC. We stopped Ceftriaxon. Still fever didn't respond and on the very next day fever was $106^{\circ} \mathrm{F}$. On $14^{\text {th }}$ day of her admission, CT scan of whole abdomen revealed mild hepatomegaly, cholelithasis, bilateral mild pleural effusion, bilateral basal consolidation and no ascites, (Fig-2). On next day, test result of RA was negative but anti ds DNA antibody was positive with titre of $129.5 \mathrm{U} / \mathrm{mL}$.

On $16^{\text {th }}$ day of her admission, Coomb's test result was negative. Biopsy of left cervical lymph node revealed acute necrotizing lymphadenopathy (Kikuchi's disease). Later on we reviewed the slide and it showed massive

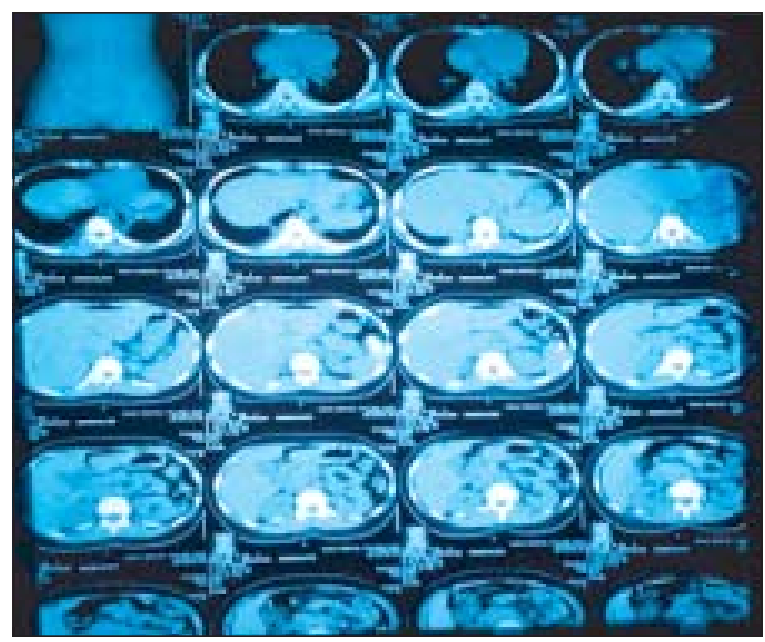

Fig.-2: CT showing bilateral pleural effusion, Hepatomegaly, basal consolidation 
apoptosis with accumulation of histiocytes, polymorphs were absent. No haematoxylin body was seen. No granuloma or malignancy was seen either. Diagnosis of histopathology report was necrotizing lymphadenopathy and differntial diagnosis was SLE lymph adenitis. (Fig-3) Complement C3, C4 and repeat urine R/M/E was normal. Repeat complete blood count showed, Hb- 8.4 gm/dl, total count of WBC- $410^{3} / \mathrm{mm}^{3}$, N-70\%, L-20\%, M-8\%, E-2\%, platelet count- $22010^{3} / \mathrm{mm}^{3}$ and ESR-100 $\mathrm{mm}$ in $1^{\text {st }}$ hour . Serum lactate dehydrogenase was 426U/L.

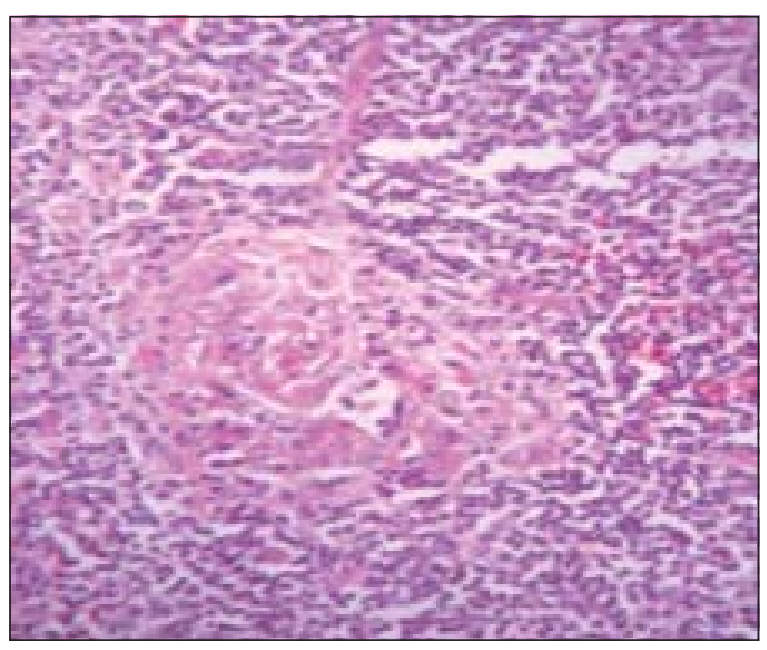

Fig-3: LN biopsy showing apoptotic necrosis.

Systemic steroid $1 \mathrm{mg} / \mathrm{kg}$ body weight with supportive treatment was started on day 20 and within 48 hours complete remission of fever occurred. Tapering of steroid was started after 2 weeks of commencement. Patient responded quickly. We discharged the patient with advised to follow up regularly. Follow up visit at $3^{\text {rd }}$ and $6^{\text {th }}$ week revealed marked clinical improvement. January 2014, she developed typical butterfly rashes on the face over the cheek, nasal bridge, sparing the nasolabial fold that are fixed, erythematous, some are flat, some are raised that are aggravated on exposure to sunlight. Also erythematous rash present over the chin. Steroid was adjusted and patient responded partially with treatment. With above regimen, we added Tab.Hydroxycholoroquine 200 mg twice daily. Then dose was adjusted accordingly. Now patient is on systemic steroid (7.5 mg) in every alternate day and hydroxychloroquine (100mg), 12 hourly. With this treatment her rashes almost disappear and she performs her daily activity normally.

\section{Discussion:}

KFD is a rare but recognized cause of pyrexia and lymphadenopathy of an unknown origin. Based on a study done by Kucukardali et al. in 2007, 330 cases of KFD were reported in the Medline database; among those, $77 \%$ were females with a mean age of 25 , and $70 \%$ of patients were under age of 30 . Asia had the most reported cases followed by Europe and America. The most common symptoms were persistent fever (35\%) and lymphadenopathy (100\%). High sedimentation rate (40\%), leucopenia (43\%), anaemia (23\%) is also observed in a majority of patients, and positive ANA has also been reported in KFD patients ${ }^{6}$. On the other hand SLE also present with pyrexia of unknown origin and lymphadenopathy. In our case, empirical antibiotic was given as a presumptive undetermined infection. Clinically, tuberculous lymphadenitis always remains a major differential diagnosis, especially in developing countries like Bangladesh. So subsequently, when we got granulomatous inflammation on FNAC of left supraclavicular lymph node, we started empirical anti TB therapy although the cytology revealed no caseous necrosis.

Histologically, SLE and KFD can be very challenging to differentiate and at times impossible. In KFD, histopathological findings include varying degrees of necrosis, histiocytic proliferation with activated $\mathrm{T}$ lymphocytes, small lymphocytes and plasma cells without granulomatous inflammation with absent neutrophils and eosinophils ${ }^{11}$. Adjacent vessels may be thrombosed. In contrast to KFD, SLE lymphadenitis demonstrates hematoxylin bodies (aggregates of degenerated nuclear debris), Azzopardi phenomenon (degenerated nuclear material aggregated in the walls of blood vessels), abundant plasma cells, prominent reactive follicular hyper-plasia, sparse cytotoxic T cells and capsular and pericapsular inflammation ${ }^{11,12}$. In our patient's biopsy specimens, massive apoptosis with accumulation of histiocyte, absence of polymorphs, granulomas and malignancy or hematoxylin bodies support the diagnosis of KFD rather than SLE. But these striking features might not be identified in every case of SLE associated lymphadenitis, however, and the diagnosis cannot always be ruled out on histological grounds alone ${ }^{13}$. Imamura and coworkers ${ }^{14}$ hypothesized that KFD might reflect a self limited SLElike autoimmune condition induced by virus infected 
transformed lymphocytes. Yet the results of serologic studies testing antinuclear antibodies, rheumatoid factor, and other immunologic parameters consistently have been negative in these patients ${ }^{15}$.

The clinical and immunological features required for diagnosis of SLE are well documented and specific. Our case fulfilled four out of the eleven ARA criteria, compatible with the diagnosis of SLE having two episodes of lymphopenia $\left(<1500 / \mathrm{mm}^{3}\right)$, highly positive ANA, anti ds DNA antibody and bilateral pleural effusion. Though there was bilateral basal consolidation on CT chest, it can be explained with SLE as a feature of bronchopneumonia.

Conclusion: Although the incidence of KikuchiFujimoto disease is rare, this disorder must be considered among the differential diagnosis when a young female patient present with fever and cervical lymphadenopathy. Clinically Kikuchi's disease may mimic systemic lupus erythomatosus. Therefore a careful histopathological examination and concomitant immunological investigations are necessary in arriving at diagnosis.

\section{Acknowledgement:}

Conflicts of Interest: None declared

\section{References:}

1. Nazarinia MA, Ghaffarpasand F, Shamsdin A, Karimi AA, Abbasi N, Amiri A. Systemic lupus erythematosus in the Fars Province of Iran. Lupus. 2008; 17:221-227.

2. Cervera R, Khamashta M, Hughes G. The Euro-lupus project: epidemiology of systemic lupus erythematosus in Europe. Lupus. 2009;18:869-874.

3. Anolik JH. B cell biology and dysfunction in SLE. Bull NYU Hosp Jt Dis 2007; 65:182-186.
4. M. Kikuchi. Lymphadenitis showing focal reticulum cell hyperplasia with nuclear debris and phagocytes. Acta haematologica Japonica.1972; 35: 379-380.

5. Y.Fujimoto,Y. Kojima, K. Yamaguchi. Cervical Sub acute necrotizing lymphadenitits. Naika.1972; 20: 920-927.

6. Y.Kucukardali, E. Solmazgul, E. Kunter, O. Oncul, S. Yildirim, and M. Kaplan. Kikuchi-fujimoto disease: analysis of 244 cases. Clinical Rheumatology. 2007;26(1): 50-54.

7. A. Hrycek, P. Cieœlik, W. Szkróbka, and J. Pajak. KikuchiFujimoto disease: a case report. Rheumatology International.2005; 26(2):179-181.

8. E. M. Bailey, N. C. Klein, and B. A. Cunha. Kikuchi's disease with liver dysfunction presenting as fever of unknown origin. The Lancet.1989; 2(8669): 986.

9. Rowell NR, Goodfield MJ. The 'Connective tissue diseases'. In : Champion RH, Burton JL, Burns DA, Breathnach SM, editors. Textbook of dermatology. 6th edn. Oxford: Blackwell Science; 1998: 2438-99.

10. El-Ramahi KM, Karrar A, Ali MA. Kikuchi’s disease and its association with systemic lupus erythematosus. Lupus. 1994; 3:409-11.

11. Hu, S. Kuo, T.T, Hong, H.S. Lupus lympha-denitis simulating Kikuchi's lymphadenitis in patients with systemic lupus erythematosus, A clinicopathological analysis of six cases and review of the literature. Pathol-ogy International.2010; 53, 221-226.

12. Hutchinson, C.B and Wang, E. Kikuchi-Fujimoto disease. Archives of Pathology \& Laboratory Medicine.2010; 134: 289-293.

13. Tsang WYW, Chan JKC, Ng CS. Kikuchi’s lymphadenitis: a morphologic analysis of 75 cases with special reference to unusual features. Am J Surg Pathol.1994;18:219-231.

14. Imamura $\mathrm{M}$, Ueno $\mathrm{H}$, Matsuura $\mathrm{A}$, et al. An ultrastructural study of subacute necrotizing lymphadenitis. Am J Pathol.1982; 107:292-299.

15. Dorfman RF. Histiocytic necrotizing lymphadenitis of Kikuchi and Fujimoto [editorial]. Arch Pathol Lab Med. 1987; 111:1026-1029. 